\title{
Quantum Hall effect in a one-dimensional lateral superlattice: Nearly dissipationless transport across high potential barriers
}

\author{
G. Müller, * D. Weiss, and K. von Klitzing \\ Max-Planck-Institut für Festkörperforschung, D-70569 Stuttgart, Germany \\ P. Středa \\ Institute of Physics, CZ-162 00 Praha, Czech Republic \\ G. Weimann \\ Walter-Schottky-Institut, Technische Universität München, D-85748 Garching, Germany
}

(Received 23 December 1994)

\begin{abstract}
We have investigated strongly modulated one-dimensional lateral superlattices in the quantum Hall regime. Although the modulation amplitude is comparable to the Fermi energy in the system, giving rise to zero magnetic field square resistances of up to $75 \mathrm{k} \Omega$, we observe nearly dissipationless transport across the barriers at integer filling factors. While the Hall resistance displays quantized plateaus, there are no gaps in the Landau level density of states. The experimental findings can be explained in terms of Büttiker's edge channel model involving the high aspect ratio of the barriers.
\end{abstract}

One-dimensional (1D) lateral superlattices fabricated from high mobility two-dimensional electron gases (2DEG) have been the subject of considerable experimental and theoretical work. In most of these systems the amplitude of the superimposed superlattice potential is small compared to the Fermi energy in the unpatterned 2DEG. The longitudinal magnetoresistance at low magnetic fields $B$ is dominated by $1 / B$-periodic commensurability oscillations. These oscillations stem from the commensurability between the two independent lengths of the system, the cyclotron radius (at the Fermi energy) $R_{c}$, and the period $a$ of the lateral superlattice. $^{1-3}$ With increasing modulation strength a quenching of these commensurability oscillations has been reported for a zero field sheet resistance of $2.6 \mathrm{k} \Omega{ }^{4}$ When the modulation strength is further increased a giant negative magnetoresistance appears when the Fermi energy of the 2DEG becomes comparable to the modulation potential. ${ }^{5}$ Little is known, however, about the transport properties of a strongly modulated 1D lateral superlattice under quantum Hall effect conditions where transport takes place across stripes with different filling factors $\nu$, alternating on a nanoscale. While the longitudinal resistance across broad stripes (100 $\mu \mathrm{m}$ scale) with different $\nu$ 's is given by the difference of the Hall resistances ${ }^{6,7}$ we find the surprising result that transport across nanoscale stripes of different carrier density can be nearly dissipationless.

Here, we study a lateral superlattice with a period $a$ of $500 \mathrm{~nm}$. The structure is designed such that the transport is ballistic across the stripe with the higher filling factor, called the well region. In the other stripe, denoted as barrier region, the carrier density is estimated to be reduced by a factor of 27 , giving rise to a substantial potential step at the interfaces between well and barrier. ${ }^{5}$ In the barrier region scattering is significantly enhanced.

We used a conventional high mobility GaAs$\mathrm{Al}_{x} \mathrm{Ga}_{1-x} \mathrm{As}$ heterostructure with a thin GaAs cap layer on top to fabricate the superlattices. The carrier density of the unpatterned material was $2.4 \times 10^{15} \mathrm{~m}^{-2}$ with a mobility of $70 \mathrm{~m}^{2} / \mathrm{V} \mathrm{s}$ at liquid helium temperatures, corresponding to a mean free path of $\sim 5.6 \mu \mathrm{m}$. The 1D superlattice was fabricated using holographic lithography. ${ }^{8}$ Two interfering laser beams of an Ar-ion laser define a grating pattern with a period of $500 \mathrm{~nm}$ which is transferred onto the 2DEG by selective etching. We use $\mathrm{H}_{2} \mathrm{O}_{2}$ buffered with $\mathrm{NH}_{4} \mathrm{OH}$ to $p \mathrm{H} 7$ at $0{ }^{\circ} \mathrm{C}$ to remove stripes from the GaAs cap layer [Fig. 1(b), upper inset]. After patterning, a Hall bar geometry was defined by conventional photolithographic techniques. The grating, oriented perpendicular to the Hall bar, covers the whole area [Fig. 1(b), lower inset]. The experiments were carried out in a ${ }^{3} \mathrm{He}$ cryostat at $T \sim 500 \mathrm{mK}$. We determined the resistivities $\rho_{x x}$ and $\rho_{x y}$ in a magnetic field normal to the 2DEG by applying a dc current of $100 \mathrm{nA}$ between contacts 1 and 2 and measuring the voltage drop between the contacts 3 and 4 (longitudinal four-point resistance $R_{12,34}$ ) or between contacts 4 and 5 (Hall resistance $\rho_{x y}=R_{12,45}$ ) with a nanovoltmeter.

Figure 1(a) displays $\rho_{x x}=R_{12,34} /(l / w)$ as a function of the magnetic field $B$. Here, $l$ is the distance between the voltage probes and $w$ is the width of the Hall bar as is sketched in the inset of Fig. 1(b). The symmetry between both magnetic field directions demonstrates the homogeneity of our device. In spite of the huge zero field resistivity of $\rho_{x x}(B=0)=74 \mathrm{k} \Omega-2000$ times larger than that of the unpatterned sample - quantum oscillations emerge at about $1 \mathrm{~T}$. As in a conventional 2DEG, the Hall resistance $\rho_{x y}$ [Fig. 1(b) ] is quantized (within the experimental accuracy) in units of $h / e^{2}$ whenever $\rho_{x x}$ displays pronounced minima. Although the height of the barriers is close to the Fermi energy $E_{F}$ in the system ${ }^{5}$ we observe nearly dissipationless transport across the superlattice.

In order to understand this peculiar behavior we first provide a rough estimate of the height of our built-in periodic potential $V(x)$ sketched in Fig. 2(a). Assume that our system 

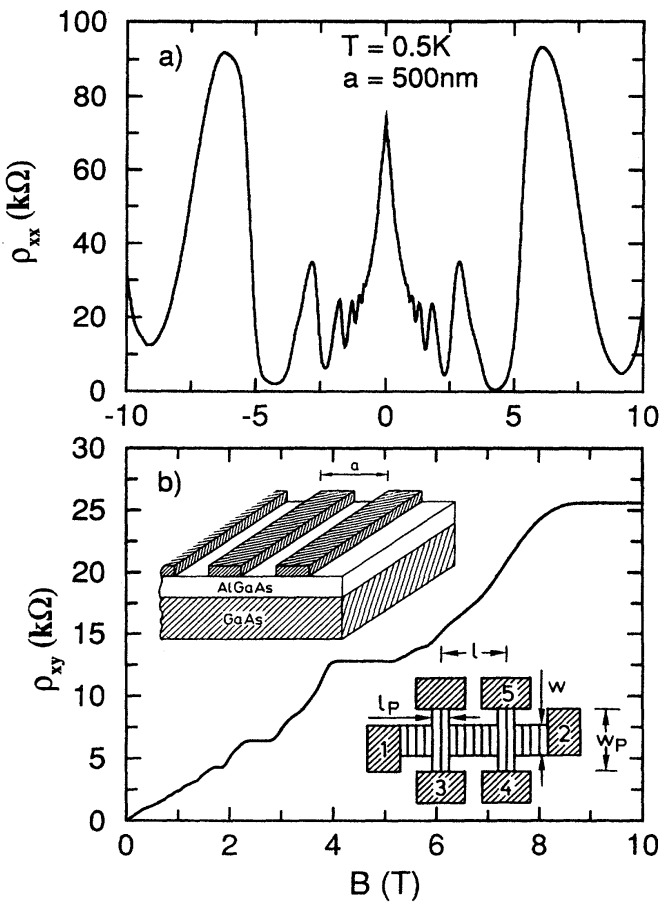

FIG. 1. $\rho_{x x}$ (a) and $\rho_{x y}$ (b) for a strongly modulated lateral superlattice for current flow perpendicular to the grating. Note the large $\rho_{x x}$ values. Upper inset: Sketch of the lateral superlattice. Lower inset: Hall bar geometry containing the lateral superlattice with $a=500 \mathrm{~nm}$ and $w=300 \mu \mathrm{m}$.

consists of alternating stripes with different but constant carrier density. Further we assume for the moment that transport in each of the stripes is diffusive and that the components of the electric field $\left(E_{x}^{l}, E_{x}^{h}, E_{y}^{l}=E_{y}^{h}\right)$ and the current density $\left(j_{x}^{l}=j_{x}^{h}, j_{y}^{l}, j_{y}^{h}\right)$ are constant within these stripes. Here, the superscripts $l$ and $h$ denote quantities in the low density region (potential barrier) and the high $n_{s}$ region (potential well), respectively. The expression for the total resistivity $\rho_{x x}$ at $B=0$ is then given within the Drude picture by ${ }^{9}$

$$
\rho_{x x}(B=0)=\frac{1}{2 e}\left(\frac{1}{n^{h} \mu^{h}}+\frac{1}{n^{l} \mu^{l}}\right) .
$$

Here $\mu^{h}, \mu^{l}$, and $n^{h}, n^{l}$ are the mobility and the carrier density in the high and low carrier density regions, respectively. For $\mu^{h}$ we take the mobility of the unpatterned reference sample $\left(\mu^{h}=70 \mathrm{~m}^{2} / \mathrm{V} \mathrm{s}\right)$. From the Shubnikov-de Haas type of oscillations in $\rho_{x x}$ [Fig. 1(a)] we deduce a carrier density $n^{h}$ of $2.4 \times 10^{15} \mathrm{~m}^{-2}$. We ascribe this carrier density to the high density stripes since it corresponds to the one measured in the unpatterned reference sample. Using the zero-field resistivity of $74 \mathrm{k} \Omega$, we find from Eq. (1) that $n^{l} \mu^{l} \sim 4.2 \times 10^{13} \mathrm{~V}^{-1} \mathrm{~s}^{-1}$. In a $2 \mathrm{DEG}$ the mobility depends on the carrier density and usually $\mu \propto n^{\gamma}$ with $\gamma=1.1-1.7$ holds. ${ }^{10}$ Using $\gamma=1.5$, we obtain $n^{l} \sim 8.7 \times 10^{13} \mathrm{~m}^{-2}$ and $\mu^{l} \sim 0.48 \mathrm{~m}^{2} / \mathrm{V} \mathrm{s}$ which corresponds to an electron mean free path of $\sim 7 \mathrm{~nm} \ll a$ in the barrier region. From the difference in the carrier density of the high a)

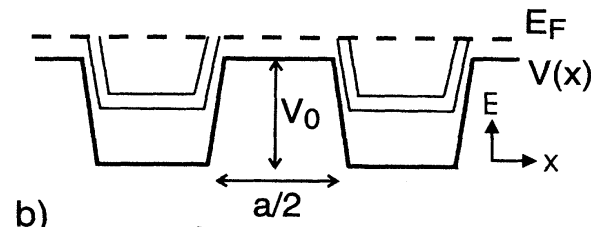

b)

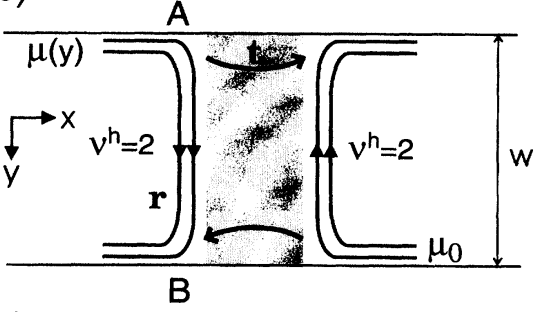

c)

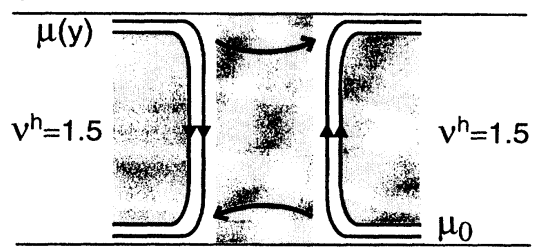

FIG. 2. (a) Conduction band edge (thick solid line) and the two lowest (spin resolved) Landau bands (thin solid lines) in a onedimensional lateral superlattice (schematic). $E_{F}$ denotes the Fermi energy. (b) Edge channels for $\nu^{h}=2$ approaching the barrier region of the lateral superlattice. The finite conductivities $\sigma_{x x}$ and $\sigma_{y y}$ in the barrier region are indicated by the shaded area. The transmission with transmission coefficient $t=1-r$ across the barrier is illustrated by the arrows. Our sketch is not to scale: in experiment $w /(a / 2) \sim 1200$. Electrons in the edge channels running from $A$ to $B$ are transferred across the barrier before they reach $B$. (c) As (b), but for $\nu^{h} \sim 1.5$. Now, backscattering can take place in the high density regions as is indicated by the shaded area.

and the low density stripes we estimate the height of the built in periodic potential $V_{0}$ to be $8.2 \mathrm{meV}$, nearly equal to $E_{F}=8.5 \mathrm{meV}$.

This rough estimate ignores the fact that transport is ballistic across the superlattice wells. Therefore $V_{0}$ has to be determined in a system which consists of alternating ballistic (mean free path $\left.\ell^{h} \gg a / 2\right)$ and diffusive $\left(\ell^{l} \ll a / 2\right)$ stripes. Corresponding model calculations for this device 5 agree remarkably with our rough estimate, also resulting in a barrier height of $0.96 E_{F}$. Within these model calculations, the giant negative low field magnetoresistance can be understood as consequence of magnetic breakdown with $\vec{k}$-dependent scattering and with anisotropic dynamics of electron states at the (modified) Fermi surface.

Why is electric transport across such an extremely modulated structure, consisting of ballistic and diffusive stripes in series, nearly dissipationless, i.e., why does $\rho_{x x}$ display minima which are close to zero? This behavior is remarkable since it is well known that the resistance measured across wide barriers is finite. For a system consisting of alternating wide stripes with (integer) filling factors $\nu^{l}$ and $\nu^{h}$, the longitudinal four-point resistance $R_{12,34}$ is given by 


$$
R_{12,34}=N \frac{h}{e^{2}}\left(\frac{1}{\nu^{l}}-\frac{1}{\nu^{h}}\right)
$$

with $N$ the number of the low density stripes. This longitudinal resistance is simply the difference of the Hall resistances of the stripes times the number of barriers. In our experiments $N$ is $\sim 900$. Hence, Eq. (2) predicts a resistance value of the order of $\sim 900 \times h / e^{2}$ for the $\rho_{x x}$ minima and cannot explain our observation.

Here, however, the barriers are not wide; their width is only $\sim 250 \mathrm{~nm}$. In Fig. 2(a) we sketch the Landau bands in an idealized lateral superlattice potential. Since the superlattice period is much larger than the magnetic length $l_{B}=\sqrt{\hbar / e B} \sim 12 \mathrm{~nm}$ at $B=4.3 \mathrm{~T}$, the Landau bands should follow the built-in modulation potential. At $4.3 \mathrm{~T}$ where $\rho_{x x}$ exhibits dissipationless transport [Fig. 1(a)] two Landau bands are occupied in the high density region (filling factor $\nu^{h}=2$ ) while in the barrier region $E_{F}$ lies within the lowest spin-split Landau level $\left(\nu^{l}<1\right)$. In the barrier region enhanced scattering ${ }^{5}$ broadens the Landau levels. In contrast to an infinite homogeneous 2DEG there are no gaps in the density of states. In spite of that transport is nearly dissipationless.

This peculiar result can be understood using Büttiker's edge channel picture. ${ }^{11}$ Within this model, transport in the quantum Hall regime is described in terms of onedimensional states. These are located at the sample boundaries where the bent-up Landau levels cross $E_{F}$. The number of edge channels is given by the filling factor $\nu$. Classically, these states correspond to skipping orbits moving along the sample boundary. While screening was not taken into account in the original model, "edge electrostatics" with perfect screening results in incompressible and compressible stripes, ${ }^{12,13}$ corresponding to the edge channels. In the edge channel picture a finite resistance is due to backscattering of the electrons from one side of the Hall bar to the other. We will show below that a "leaky" barrier effectively prevents backscattering which we believe is the origin of the nearly dissipationless transport. In Fig. 2(b) the edge channels are shown schematically for filling factor $\nu^{h}=2$ in the high density region. Consider a situation where the edge channels on the left-hand side of the barrier in Fig. 2(b) have a chemical potential $\mu(y)>\mu_{0}$, where $\mu_{0}$ is the chemical potential of the approaching edge channels on the right-hand side. In the barrier region states of the lowest Landau level are located at the Fermi energy and hence the corresponding conductivities in the barrier, $\sigma_{x x}$ and $\sigma_{y y}$, are finite at $\nu^{h}=2$. If there is a chemical potential difference across the barrier, electrons will flow from the left side of the barrier to the right side. In the following we assume sufficiently strong interedge channel scattering ${ }^{14-17}$ between the two neighboring edge channels running from $A$ to $B$, so that they have the same $y$-dependent chemical potential $\mu(y)$. Further we assume that the decrease of the chemical potential $\mu$ of electrons on their way from $A$ to $B$ is described by $d \mu / d y$ $=-P\left[\mu(y)-\mu_{0}\right]$, giving rise to a reflection coefficient of $r=\exp (-P w)$ for a single barrier. Here, $w$ is the length of a barrier, equal to the width of our Hall bar [see inset of Fig. 1 (b)]. The resulting longitudinal resistance $R_{12,34}$ for $N$ barriers is

$$
R_{12,34}=N \frac{h}{e^{2} \nu^{h}} \frac{1}{\exp (P w)-1} .
$$

When we neglect the barriers within the region of the potential probes [Fig. 1(b), lower inset] we can estimate $P$ from $R_{12,34}$ at filling factor $\nu^{h}=2$ : With the aspect ratio of $l / w=1.5, R_{12,34}=1.5 \times \rho_{x x} \sim 1.9 \mathrm{k} \Omega$ (averaged over $\pm B$ ). With a number of 700 barriers in between the voltage probes the single barrier resistance for $\nu^{h}=2$ is only $\sim 2.7 \Omega$. For $w=300 \mu \mathrm{m}$ we obtain $P^{-1}=35 \mu \mathrm{m}$, and $r=2.1 \times 10^{-4}$.

In Fig. 2(b) an electron entering at $A$ can either run along the barrier (reflected edge channel) or use the finite conductivity in the barrier region to be transmitted through the 250 $\mathrm{nm}$ wide barrier. If reflected, the electrons run along the 300 $\mu \mathrm{m}$ long barrier. Since the barriers have a finite transmission, the edge channel will "leak" to the other side of the barrier and will return to the same edge of the Hall bar. The simple model above shows, that on the average an electron is transmitted through the barrier after $35 \mu \mathrm{m}$, hence long before it reaches the other side of the Hall bar. This is the reason why there is no backscattering along barrier. In spite of the finite conductivity inside the barrier, there is essentially no backscattering, too. This is due to the huge aspect ratio of a single barrier of about 1200 (barrier width $\approx 250 \mathrm{~nm}$, barrier length $\approx 300 \mu \mathrm{m}$ ). Consequently, transport is nearly dissipationless.

Calculations within a classical trajectory network model agree with the simple model given above. For the case of two edge channels in the high density region the resistance across a single barrier was calculated as a function of the barrier filling factor with the aspect ratio of the barrier as parameter. ${ }^{18}$ By decreasing the filling factor in the barrier region, starting at filling factor 2 , the longitudinal resistance increases continuously. If the filling factor in the barrier region becomes 1 [ $\sigma_{x x}$ (underneath the barrier) $=0$ ], the longitudinal resistance across one barrier equals $h / 2 e^{2}$. Reducing the filling factor further, can give rise to a pronounced resistance dip. The resulting resistance minimum occurs, when the Fermi energy in the barrier region coincides with the lowest Landau level $\left[\sigma_{x x}\right.$ (underneath the barrier $\left.) \neq 0\right]$. The depth of the resistance minimum with respect to the plateau depends sensitively on the aspect ratio of the barrier region. Increasing the aspect ratio results in a deeper minimum. For an aspect ratio of 10 the resistance drops by $35 \%$, for an aspect ratio of 25 the resistance drops by $70 \% .{ }^{18}$ Our simple model (aspect ratio 1200) is consistent with this theory.

Now we focus on the dissipative part of $\rho_{x x}$ in Fig. 1(a), i.e., the resistance maxima. For $B \sim 6 \mathrm{~T}$ a pronounced maximum with $\rho_{x x}=93 \mathrm{k} \Omega$ emerges. For this field the filling factor in the well region is $\nu^{h} \sim 1.5$, whereas the filling factor in the barrier is $\nu^{l}<1$. Since $\sigma_{y y} \neq 0$, backscattering across the Hall bar can take place resulting in a voltage drop along the current direction. Backscattering across the Hall bar can now take place inside the well regions. This together with the large number of barriers causes the huge resistance maxima.

The Hall resistance in Fig. 1(b) shows pronounced plateaus, quantized in units of $h / e^{2}$. This leads to the question, whether the modulation has an influence on the Hall resistance. If we model the transport within the edge channel picture we get for the Hall resistance the following expression: 


$$
R_{12,45}=\frac{h}{e^{2} \nu^{h}}\left(1+\frac{l_{p}}{a} r^{*}\right)^{-1}
$$

with $r^{*}=\exp \left(-P w_{p}\right)$. Here, $w_{p}=900 \mu \mathrm{m}$ and $l_{p}=100 \mu \mathrm{m}$ [Fig. 1(b), lower inset]. Using the experimentally determined value for $P^{-1}$ of $35 \mu \mathrm{m}$ at $\nu^{h}=2$, corresponding to the Hall plateau around $4.3 \mathrm{~T}$ in Fig. 1(b), we obtain for the correction term $l_{p} r^{*} / a \approx 1.4 \times 10^{-9}$. This estimate illustrates why no deviations from the quantized values were observable.
In summary we have demonstrated nearly dissipationless transport in strongly modulated lateral 1D superlattices in the quantum Hall regime. This effect is ascribed to the pronounced transmission probability of edge channels across the superlattice barriers, although their height is comparable to the Fermi energy. Due to the high aspect ratio of the barriers, backscattering across the Hall bar is effectively suppressed.

We thank F. Schartner, M. Riek, and S. Tippmann for their expert help in the processing of the samples.
*Present address: Philips Research Laboratories, Eindhoven, The Netherlands.

${ }^{1}$ D. Weiss, K. von Klitzing, K. Ploog, and G. Weimann, Europhys. Lett. 8, 178 (1989); R. R. Gerhardts, D. Weiss, and K. von Klitzing, Phys. Rev. Lett. 62, 1173 (1989).

${ }^{2}$ R. W. Winkler, J. P. Kotthaus, and K. Ploog, Phys. Rev. Lett. 62, 1177 (1989).

${ }^{3}$ C. W. J. Beenakker, Phys. Rev. Lett. 62, 2020 (1989).

${ }^{4}$ P. H. Beton, E. S. Alves, P. C. Main, L. Eaves, M. W. Dellow, M. Henini, O. H. Hughes, S. P. Beaumont, and C. D. W. Wilkenson, Phys. Rev. B 42, 9229 (1990).

${ }^{5}$ G. Müller, P. Středa, D. Weiss, K. von Klitzing, and G. Weimann, Phys. Rev. B 50, 8938 (1994).

${ }^{6}$ R. J. Haug, A. H. MacDonald, P. Středa, and K. von Klitzing, Phys. Rev. Lett. 61, 2797 (1988); S. Washburn, A. B. Fowler, H. Schmid, and D. Kern, ibid. 61, 2801 (1988).

${ }^{7}$ G. Müller, D. Weiss, S. Koch, K. von Klitzing, H. Nickel, W. Schlapp, and R. Lösch, Phys. Rev. B 42, 7633 (1990).

${ }^{8}$ D. Heitmann, in Electronic Properties of Multilayers and Low Dimensional Semiconductor Structures, edited by J. M. Chamberlain, L. Eaves, and J. C. Portal (Plenum Press, New York 1990), p. 151.

${ }^{9}$ Similar expressions can be found in C. Herring, J. Appl. Phys. 31,
1939 (1960).

${ }^{10}$ K. Hirakawa and H. Sakaki, Phys. Rev. B 33, 8291 (1986).

${ }^{11}$ For a review of the edge channel model, see M. Büttiker, in Nanostructured Systems, edited by M. Reed, Semiconductors and Semimetals Vol. 35 (Academic Press, Inc., San Diego, 1992), p. 191.

${ }^{12}$ D. B. Chklovskii, B. I. Shklovskii, and L. I. Glazmann, Phys. Rev. B 46, 4026 (1992).

${ }^{13}$ K. Lier and R. R. Gerhardts, Phys. Rev. B 50, 7757 (1994).

${ }^{14}$ S. Komiyama, H. Hirai, S. Sasa, and S. Hiyamizu, Phys. Rev. B 40, 12566 (1989).

${ }^{15}$ B. J. van Wees, L. P. Kouwenhoven, E. M. M. Willems, C. J. P. M. Harmans, J. E. Mooji, H. van Houten, C. W. J. Beenakker, J. G. Williamson, and C. T. Foxon, Phys. Rev. B 43, 12431 (1991).

${ }^{16}$ B. W. Alphenaar, P. L. McEuen, R. G. Wheeler, and R. N. Sacks, Phys. Rev. Lett. 64, 677 (1990).

${ }^{17}$ G. Müller, D. Weiss, A. V. Khaetskii, K. von Klitzing, S. Koch, H. Nickel, W. Schlapp, and R. Lösch, Phys. Rev. B 45, 3932 (1992); G. Müller, D. Weiss, K. von Klitzing, K. Ploog, H. Nickel, W. Schlapp, and R. Lösch, ibid. 46, 4336 (1992).

${ }^{18}$ P. Středa (unpublished); R. J. Haug, J. Kucera, P. Středa, and K. von Klitzing, Phys. Rev. B 39, 10892 (1989). 
a)
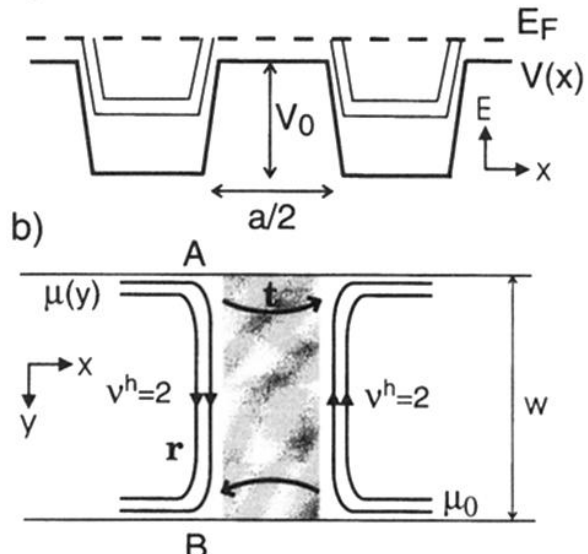

c)

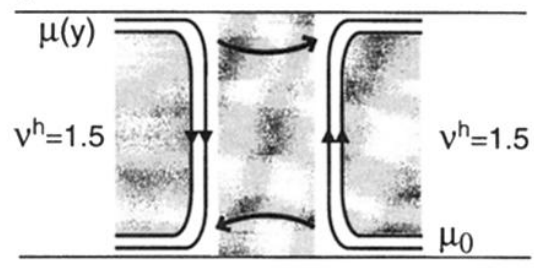

FIG. 2. (a) Conduction band edge (thick solid line) and the two lowest (spin resolved) Landau bands (thin solid lines) in a onedimensional lateral superlattice (schematic). $E_{F}$ denotes the Fermi energy. (b) Edge channels for $\nu^{h}=2$ approaching the barrier region of the lateral superlattice. The finite conductivities $\sigma_{x x}$ and $\sigma_{y y}$ in the barrier region are indicated by the shaded area. The transmission with transmission coefficient $t=1-r$ across the barrier is illustrated by the arrows. Our sketch is not to scale: in experiment $w /(a / 2) \sim 1200$. Electrons in the edge channels running from $A$ to $B$ are transferred across the barrier before they reach $B$. (c) As (b), but for $\nu^{h} \sim 1.5$. Now, backscattering can take place in the high density regions as is indicated by the shaded area. 